\title{
Development of Synaptic Arrays in the Inner Plexiform Layer of Neonatal Mouse Retina
}

\author{
LESLIE J. FISHER \\ The University of Michigan, Ann Arbor, Michigan 48109
}

\begin{abstract}
Retinas from mice of the C57BL/6 strain were sampled at frequent intervals from birth to postnatal day 33 to determine the numerical density of conventional and ribbon synapses within the inner plexiform layer (IPL) as a function of time. Synaptic arrays of the IPL were formed in three phases. During Phase I, from day 3 to day 10 , conventional synapses were produced at a mean rate of 0.44 synapses $/ 1,000 \mu \mathrm{m}^{3} /$ hour, but no ribbons were seen. During Phase II, from day 11 to day 15 , ribbons formed at a rate of 0.38 ribbons $/ 1,000$ $\mu \mathrm{m}^{3} /$ hour and conventional synapses were produced at a rate of 1.15 synapses/ $1,000 \mu \mathrm{m}^{3} /$ hour. Phase III began at day 15 , the approximate time of eye opening in these animals, and was characterized by a sharp reduction in the rate of production of both ribbons and conventional synapses. During this phase ribbons achieved a final mean density of 113 ribbons $/ 1,000 \mu \mathrm{m}^{3}$ and conventionals achieved a final mean density of 250 synapses $/ 1,000 \mu \mathrm{m}^{3}$. Serial synapses appeared in Phase II but remained at low densities.
\end{abstract}

The retinal inner plexiform layer (IPL) is particularly suitable for quantitative analysis because of its precisely defined borders, its involvement with only three presynaptic neuronal elements, and the ability to distinguish ribbon from conventional synaptic complexes. Ribbon synapses in the IPL are unique to bipolar cells while symmetrical or asymmet. rical conventional synapses are found only in the processes of amacrine cells (Dowling and Boycott, '66; Raviola and Raviola, '67; Witkovsky and Dowling, '69) or interplexiform cells (Dowling et al., '76; Kolb and West, '77). Furthermore, serial synapses can be identified as a subset of conventional synapses in which a process is simultaneously postsynaptic to a conventional synapse from an amacrine cell or an interplexiform cell and presynaptic to a bipolar, amacrine, interplexiform, or ganglion cell (Dowling and Boycott, '66; Dubin, '70; Kolb and West, '77). This ability to precisely identify the input and interneuronal connections allows a detailed analysis to be made of the temporal and spatial patterns of connectivity within the neuropil. The purpose of this paper is to describe quantitatively the temporal formation of synaptic arrays in the mouse from an undifferentiated state at birth until the prepubertal period at 33 days of age.

\section{MATERIALS AND METHODS}

A colony of the inbred strain of C57BL/6 mice originally purchased from Microbiological Associates was maintained under a 14-hour light, 10-hour dark cycle with food (Purina Rat Chow) and water supplied ad libitum.

After random mating, pregnant females were segregated and kept in separate cages with their litters. Daily inspections determined the day of birth (day 0). Neonates of both sexes were studied.

Unanesthetized animals were killed by cervical dislocation at the same time of day (1300 \pm 1 hour). Eyes were sutured with 7-0 suture silk at the dorsal-most aspect - which allowed orientation during embedding and sectioning - and then excised. After hemisection and lentectomy the eyes were fixed in a combined fixative of $3 \%$ glutaraldehyde, $2 \%$ formaldehyde, $1 \%$ acrolein and $2.5 \%$ dimethyl sulfoxide in $0.1 \mathrm{M}$ sodium cacocylate buffer at $\mathrm{pH} 7.2$ for approximately 18 hours, washed in $0.1 \mathrm{M}$ cacodylate buffer and post-fixed in $2 \%$ osmium tetroxide in the same buffer. Eyes were stained en bloc with $0.5 \%$ aqueous uranyl acetate, rinsed, dehydrated through alcohols, and embedded in Epon.

A second set of animals ( 6 adults, 8 pups- 
13 days old) were fixed by one cycle through the Golgi-Kopsch procedure reported by Colonnier ('64). The eyes were immersed for seven days each in the chromate and silver nitrate solutions. These Golgi-stained retinas were used to identify neuronal characteristics which could be used to determine cell types in the experimental retinas.

Eyes were serially sectioned at $75 \mu \mathrm{m}$ in the horizontal plane beginning at the ventralmost point and mounted onto plastic slides. The section which passed through the optic disc was located and a portion of retina within $750 \mu \mathrm{m}$ of the optic disc was mounted on a plastic blank for semi-thin $(1 \mu \mathrm{m})$ and thin (600-700 A) sectioning. All electron micrographs were taken between $150 \mu \mathrm{m}$ and 500 $\mu \mathrm{m}$ from the optic nerve.

Nuclei were counted and layer thicknesses were measured on semi-thin sections stained with methylene blue. Nuclear densities were computed from data obtained by counting and using an Abercrombie correction (Abercrombie, '46).

Electron micrographic mosaics (final magnification $26,000 x$ ) of the entire thickness (vitreal to scleral dimension) of IPL were constructed using micrographs of thin sections stained with both uranyl acetate and lead citrate. The average area of IPL covered by one mosaic was $900 \mu \mathrm{m}^{2}$ as measured by polar planimetry.

To insure that the magnification of each mosaic was precisely known an electron micrograph of a calibration grid (Fullam No. 1002) was taken each time the magnification of the electron microscope was changed. At least one calibration micrograph was taken per session, and all were processed with the mosaics. Every mosaic, therefore, has a calibration micrograph to which it is directly related.

Ribbon, conventional, and serial-conventional synapses were identified and the lengths of their profiles were measured on the mosaics. The resulting data were reduced using a modified Abercrombie correction (Dubin, '70). Serial-conventionals were included in the data to be presented for conventionals.

The criterion used to identify a conventional synapse was the presence of at least three synaptic vesicles grouped next to a membrane specialization (fig. 1). The presence of the ribbon was the criterion for ribbon synapses (fig. 2). While most of the ribbons were associated with membrane specializations in the same plane of section, occasionally ribbons not lying near a membrane were found. The statistics reported below include ribbons both with and without an associated membrane specialization. In every instance counts of synapses and ribbons were done using a blind procedure in which the age and animal were not known until the data were reduced.

\section{Quantitative analysis}

The synapses are first defined in terms of their numerical densities (Weibel, '69) which are expressed in numbers per $1,000 \mu \mathrm{m}^{3}$. This statistic is the mean number of nuclei or synapses per unit volume of the retinal layer in question.

Statistics of developmental interest are the numbers of synapses or cells present at a given locus at a given time, and the rate of synapse formation. Retinal planimetric density and synapses per neuron expressed as a function of age supply these data.

Retinal planimetric density is computed by multiplying numerical density by thickness of the layer in question. The product is given in numbers per $1,000 \mu \mathrm{m}^{2}$ and represents the number of synapses or nuclei contained in a column which has a cross-sectional area of $1,000 \mu \mathrm{m}^{2}$ and a height equal to the thickness of the layer. The area on which these nuclei or synapses can be considered to be located is in the plane of the layer at a level midway between the layer's boundaries with the adjacent lavers. Planimetric densities, therefore, are the number of nuclei or synapses referred to a unit area in the plane of the retina. Planimetric density gives the number of synapses or nuclei which directly underlie the receptors excited by a spot of light of unit area.

The data are also expressed as the mean number of synapses per inner nuclear layer (INL) nucleus by dividing synaptic planimetric by nuclear planimetric density. Rates of ribbon and conventional synapse formation per neuron can be calculated from these statistics when plotted as a function of time.

\section{RESULTS}

\section{Golgi description}

To express the synaptic data as a function of the number of amacrine or bipolar nuclei, a reliable method of identifying the individual nuclei in the INL was needed. Accordingly, Golgi-stained retinas were analyzed to iden- 


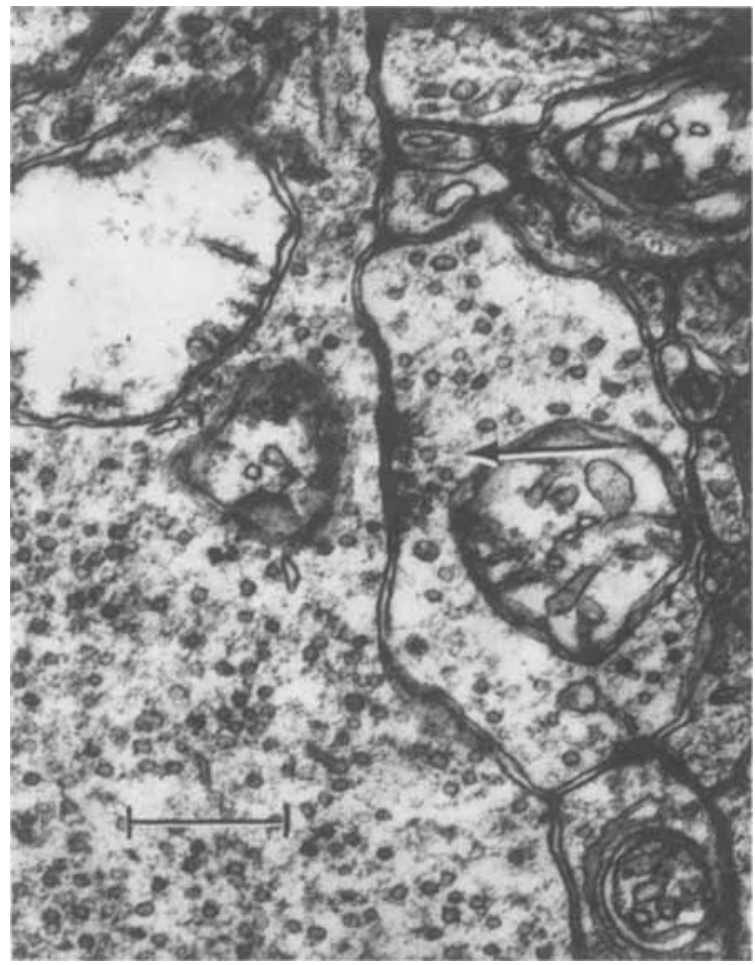

Fig. 1 Conventional synapse from the mouse IPL (arrow). Calibration Bar $=0.5 \mu \mathrm{m}$.

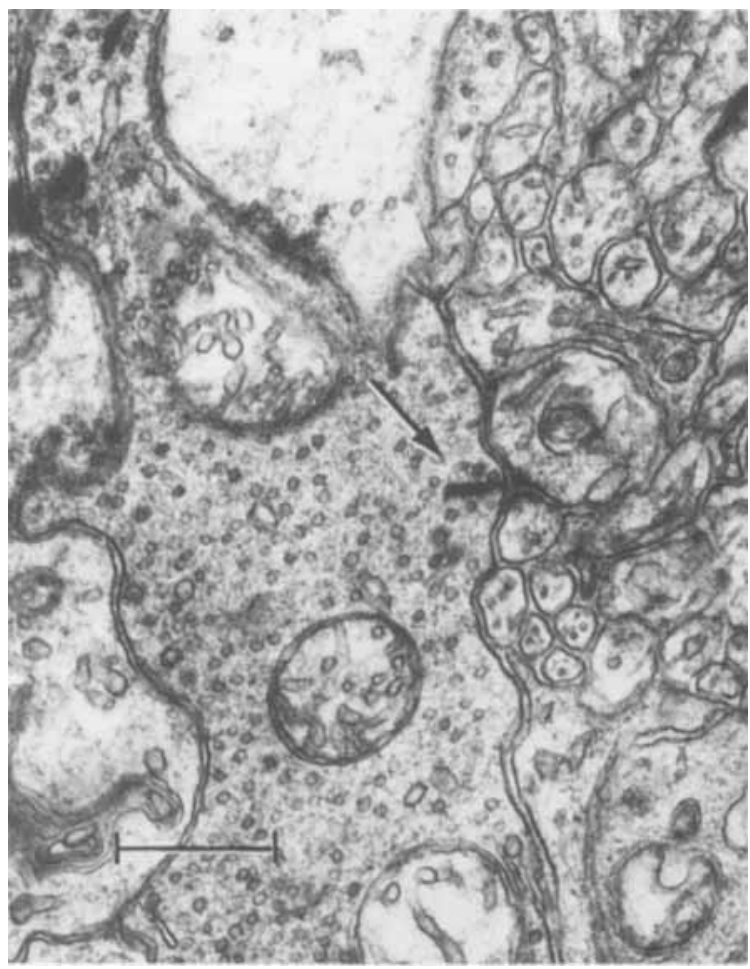

Fig. 2 Ribbon from the mouse IPL (arrow), Calibration Bar $=0.5 \mu \mathrm{m}$. 


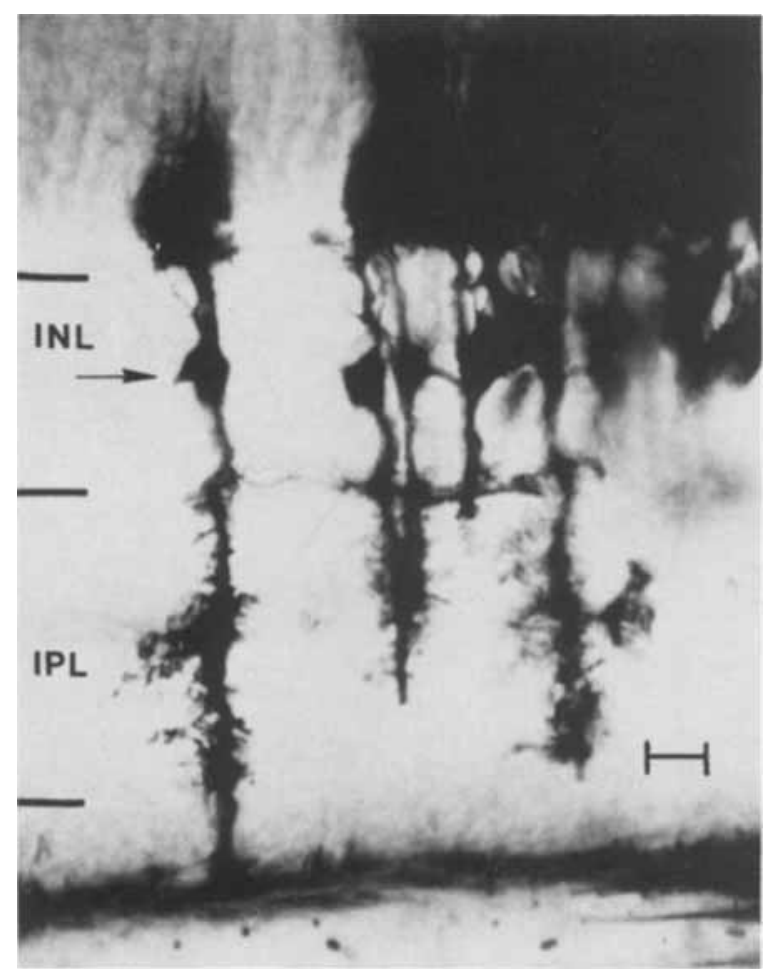

Fig. 3 Müller cells of the mouse retina. In the mouse the nuclei of Müller cells form a single stratum (arrow). INL, Inner Nuclear Layer; IPL, Inner Plexiform Layer. Golgi-Kopsch stain. Calibration Bar = $10 \mu \mathrm{m}$.

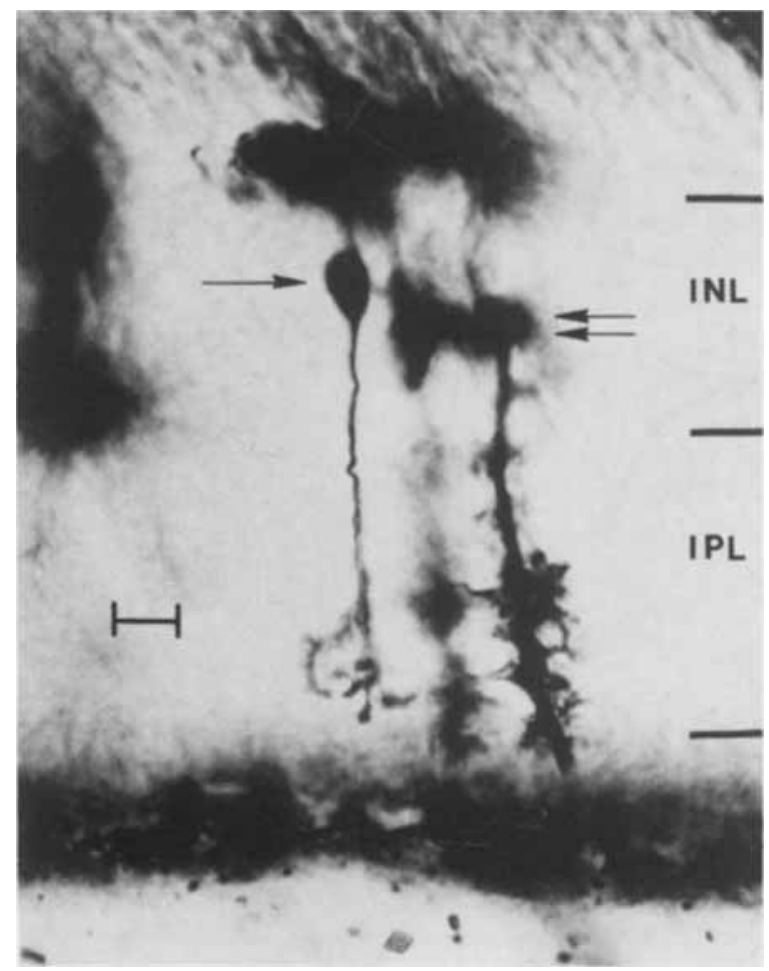

Fig. 4 Bipolar cell (single arrow) and Müller cell (double arrow) of the mouse retina as stained by the Golgi-Kopsch method. In all of the Golgi-stained material examined, bipolar nuclei were found sclerad to the level of Müller nuclei. INL, Inner Nuclear Layer; IPL, Inner Plexiform Layer. Calibration Bar $=10 \mu \mathrm{m}$. 


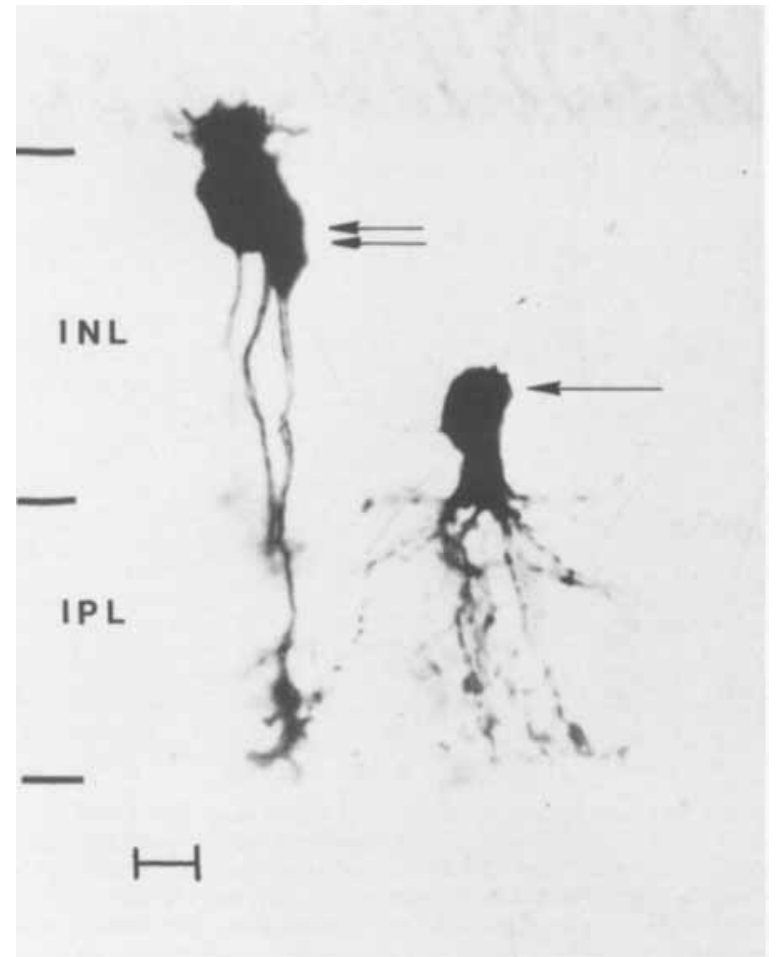

Fig. 5 Amacrine cell (single arrow) and bipolar cell (double arrow) of the mouse retina. Amacrine nuclei were always located vitread to the Müller nuclei. INL, Inner Nuclear Layer; IPL, Inner Plexiform Layer. Golgi-Kopsch stain. Calibration Bar $=10 \mu \mathrm{m}$.

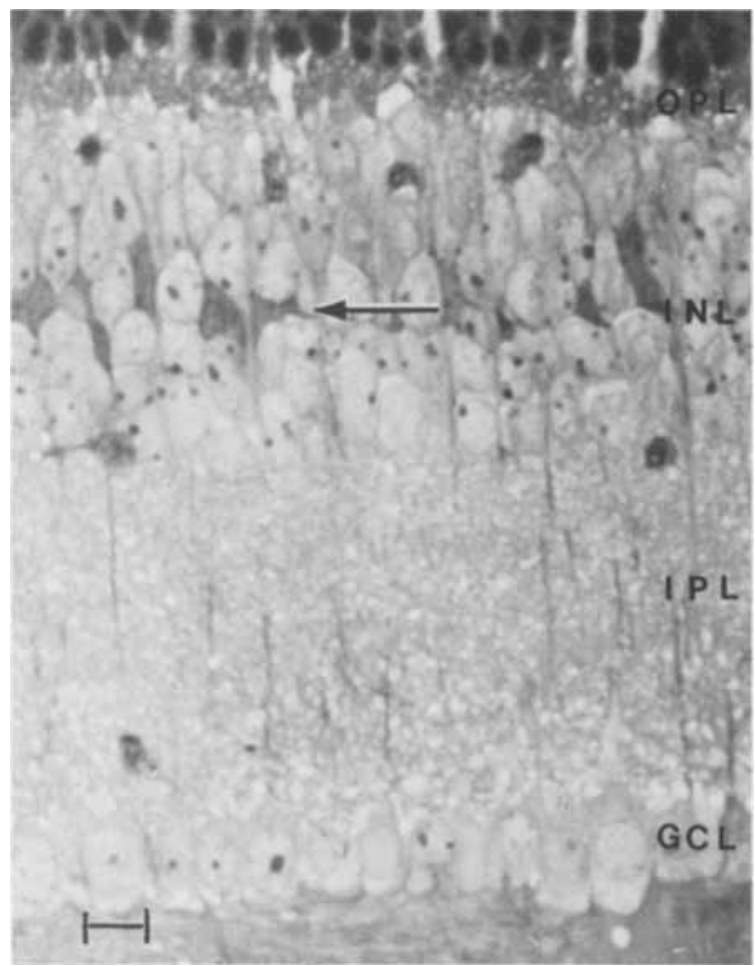

Fig. 6 Mouse retina. The stratum of darkly stained nuclei (arrow) corresponds to the Müler nuclei seen in the Golgi-stained material (fig. 3). OPL, Outer Plexiform Layer; GCL, Ganglion Cell Layer; INL, Inner Nuclear Layer; IPL, Inner Plexiform Layer. Methylene Blue stain. Calibration Bar $=10 \mu \mathrm{m}$. 


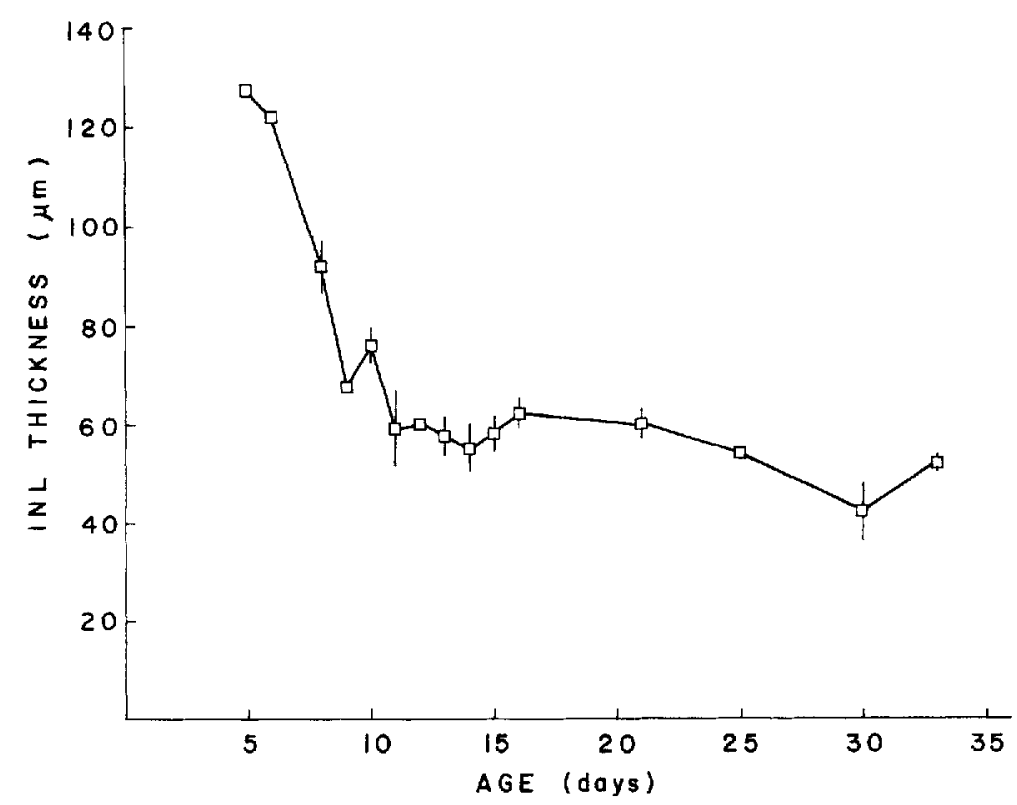

Fig. 7 Thickness of the inner nuclear layer (INL) as a function of age. Error bars in this and all subsequent figures represent \pm SEM. All thicknesses were measured between $200 \mu \mathrm{m}$ and $750 \mu \mathrm{m}$ from the optic nerve head. Measurements were made from the border between the inner plexiform layer (IPL) and INL to the scleral margin of the INL. Care was taken to insure that the most representative sections perpendicular to the surface of the retina were used for measurement. In most cases this was determined by choosing those sections with the longest outer segment length.

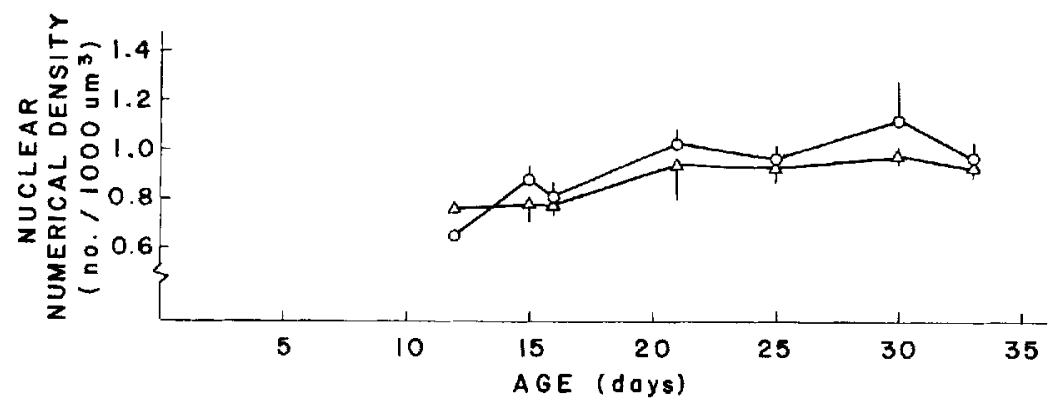

Fig. 8 Numerical densities of inner nuclear layer (INL) nuclei as a function of age. Triangles represent densities of amacrine and interplexiform nuclei within the INL; circles represent similar densities of bipolar nuclei.

tify bipolar, amacrine, interplexiform, and Müller cells and to determine criteria which could be used in their identification in conventionally stained sections. The position of the nucleus within the INL proved to be a sufficient criterion for identification of nuclei as bipolar or amacrine and interplexiform. Interplexiform cells have been seen in the mouse retina (Fisher, '79), but at a very low frequency of staining. When stained, they have been reported to be at the level of amacrine nuclei
(Boycott et al., '75; Kolb and West, '77; Fisher, '79). Accordingly, no effort was made to distinguish between amacrine and interplexiform cells in the nuclear analysis.

Figures 3-5 are light micrographs of Golgistained Müller, bipolar, and amacrine cells. More than 150 examples of each cell type were observed. Figure 6 shows a semi-thin section, at the same magnification, stained with methylene blue.

Müller cells have a distinctive appearance 


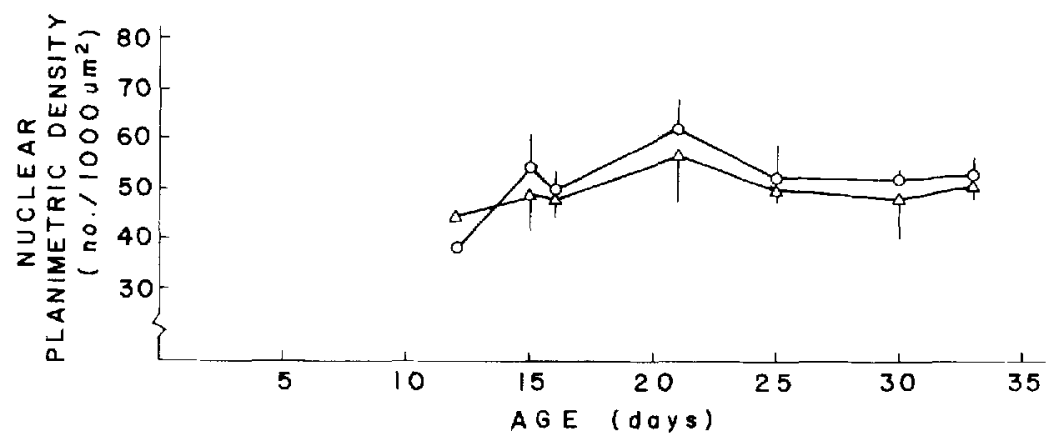

Fig. 9 Planimetric density of inner nuclear layer (INL) nuclei as a function of age. Triangles represent the density of amacrine and interplexiform nuclei, circles represent the density of bipolar nuclei. These data are the product of numerical density and INL thickness at each sampled age.

in Golgi-stained preparations (fig. 3) (Ramón y Cajal, '72). Characteristically, the convexly indented perikarya were found in the center of the INL, midway between the borders of the IPL and outer plexiform layer (OPL) (see also Polyak, '57). In thin sections stained with methylene blue dark nuclei of shape and location similar to the Golgi-stained Müller nuclei formed a well-defined stratum within the INL (fig. 6). These nuclei were then classified as Müller cells in the semi-thin sections.

Several different sub-types of bipolar and amacrine cells were observed, but all Golgistained bipolar nuclei were found sclerad to the stratum of Müller nuclei, while all Golgistained amacrine nuclei were vitread to that stratum. One cell, identified as an interplexiform cell, was found among the amacrine nuclei (Fisher, '79). All neuronal nuclei in semithin sections, therefore, were classified as either bipolar or amacrine (including interplexiform) by their position relative to the Müler nuclei. Horizontal cells, the nuclei of which were found among the bipolar nuclei, were identified in the methylene blue stained sections by the location and characteristics of the cell body. It was both larger and more lightly stained than the surrounding bipolars, and found in the most distal part of the inner nuclear layer. Since the horizontal cells do not contribute synapses to the IPL, they were identified only to exclude them from the bipolar counts and were not otherwise used in the analysis.

\section{Retinal layers}

At day 5 the inner nuclear layer consisted of many undifferentiated cells. The location of the OPL, however, was quite distinct and thus
INL thickness could easily be measured (fig. 7). Between days 5 and 15 , the INL thickness decreased by nearly $50 \%$ and stabilized at approximately $60 \mu \mathrm{m}$ at day 15 . By day 33 another slight decrease in thickness occurred.

The numerical densities of bipolar nuclei and of amacrine plus interplexiform nuclei increased slightly over days 12 through 21 and stabilized between days 21 and 33 (fig. 8). $\mathrm{Nu}$ clear planimetric density remained constant for both subsets of nuclei over the same period (fig. 9). These results indicate that there was no net change in the number of INL neurons at this retinal locus between days 12 and 33 . In retinas less than 12 days old the bipolar and amacrine nuclei could not be reliably distinguished. Nuclear counts, therefore, were started at day 12 .

Inner plexiform layer thickness was measured within $750 \mu \mathrm{m}$ of the optic nerve on days 3 to 33 (fig. 10). From day 5 the IPL thickness increased until day 15 after which it remained constant at approximately $60 \mu \mathrm{m}$.

\section{Synaptic size distributions}

The length of the membrane specialization associated with conventional synapses was measured as the chord of the arc formed by the presynaptic membrane (fig. 1). In some instances where the membrane specializations were indistinct, the width of the associated cluster of vesicles along the adjacent membrane defined the limits of the synapse. No synapses were seen before day 3 . Thereafter, the mean length was roughly constant apart from the two decreases at days 8 and 13 (fig. 11).

Ribbons enlarged as the animals aged (fig. 12). Although ribbon length cannot be corre- 


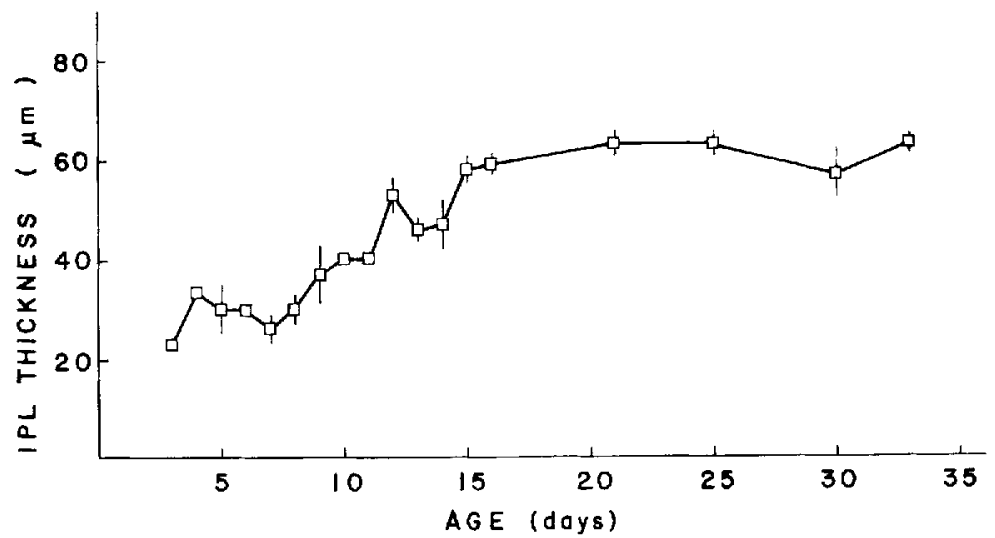

Fig. 10 Thickness of the inner plexiform layer (IPL) as a function of age. Points here are measured at the same loci as were the points in figure 7 . Measurements were taken from the border between the INL and the ganglion cell layer to the border between the IPL and INL.

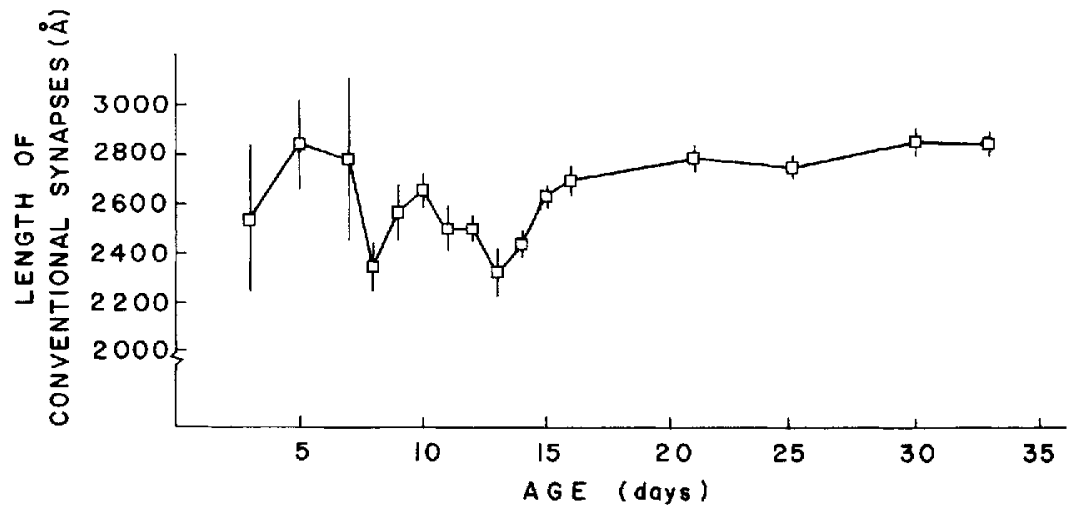

Fig. 11 Mean length of conventional synapses versus age. An average of 135 synapses are included in each data point.

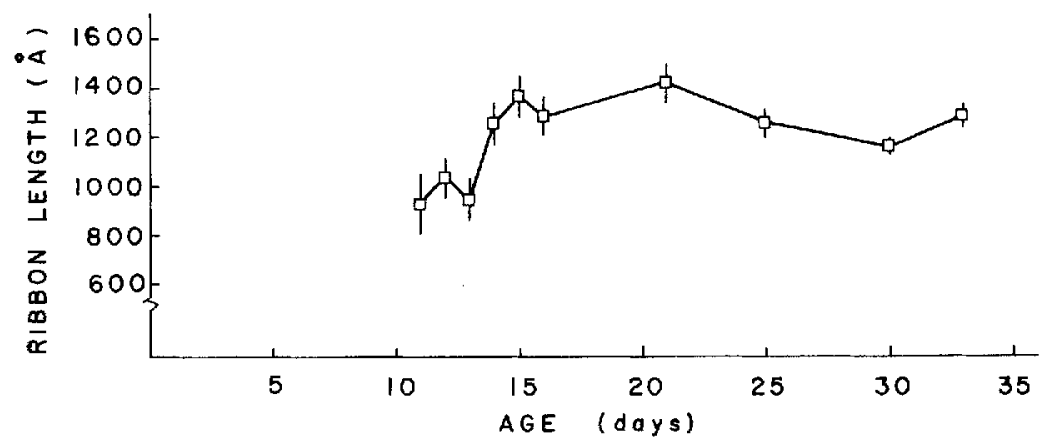

Fig. 12 Mean length of ribbons versus age. An average of 45 ribbons are included in each data point.

lated with any known synaptic functions at present, this increase in size suggests a process of maturation.

\section{Development of synaptic arrays}

The synaptic arrays developed in three phases.
Phase I (day 3 to day 10 ) was characterized oy an absence of ribbon synapses and the appearance of conventionals. During this time the thickness of the IPL increased to $40 \mu \mathrm{m}$ (fig. 10). The numerical density of conventionals increased at a mean rate of $0.44 \mathrm{syn}$. apses $/ 1,000 \mu \mathrm{m}^{3} /$ hour and reached 85 syn- 


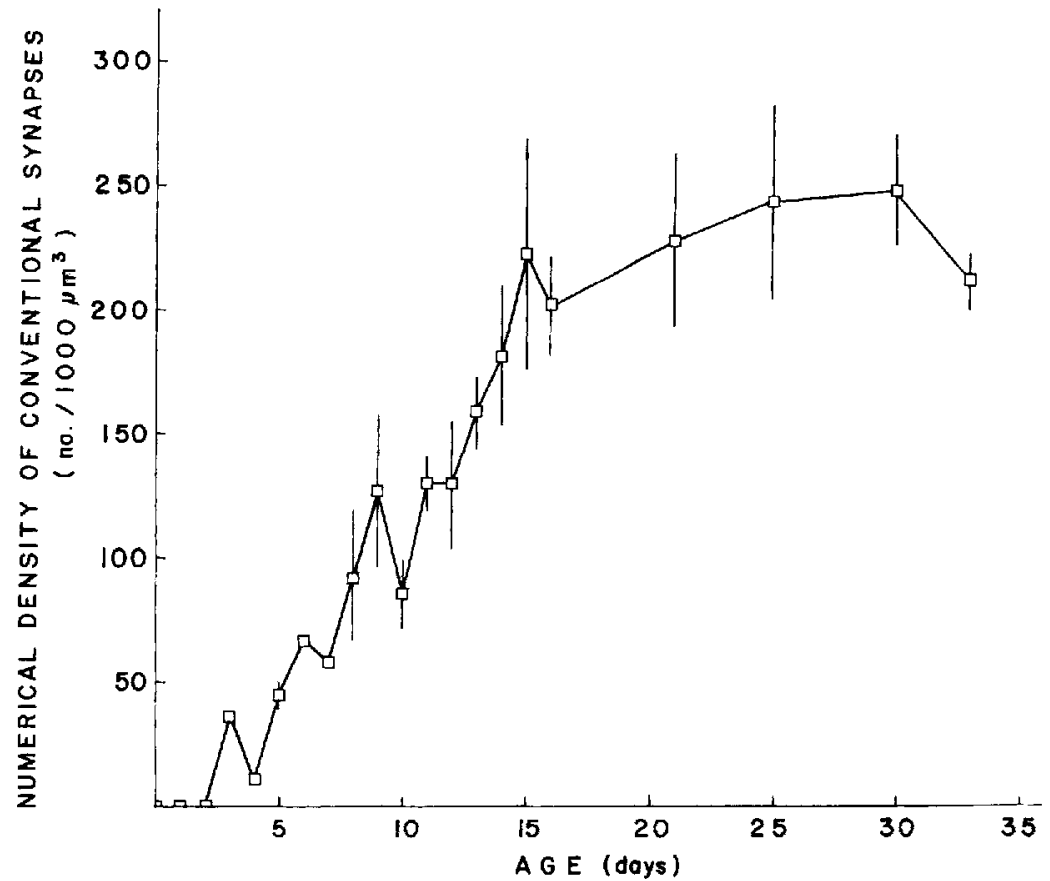

Fig. 13 Numerical density of conventional synapses versus postnatal age. Each point after day seven represents data from six animals on the average, with no point representing less than three animals. Three animals were sampled on each of days zero, one, two, and five. One animal was sampled for each of the remaining points.

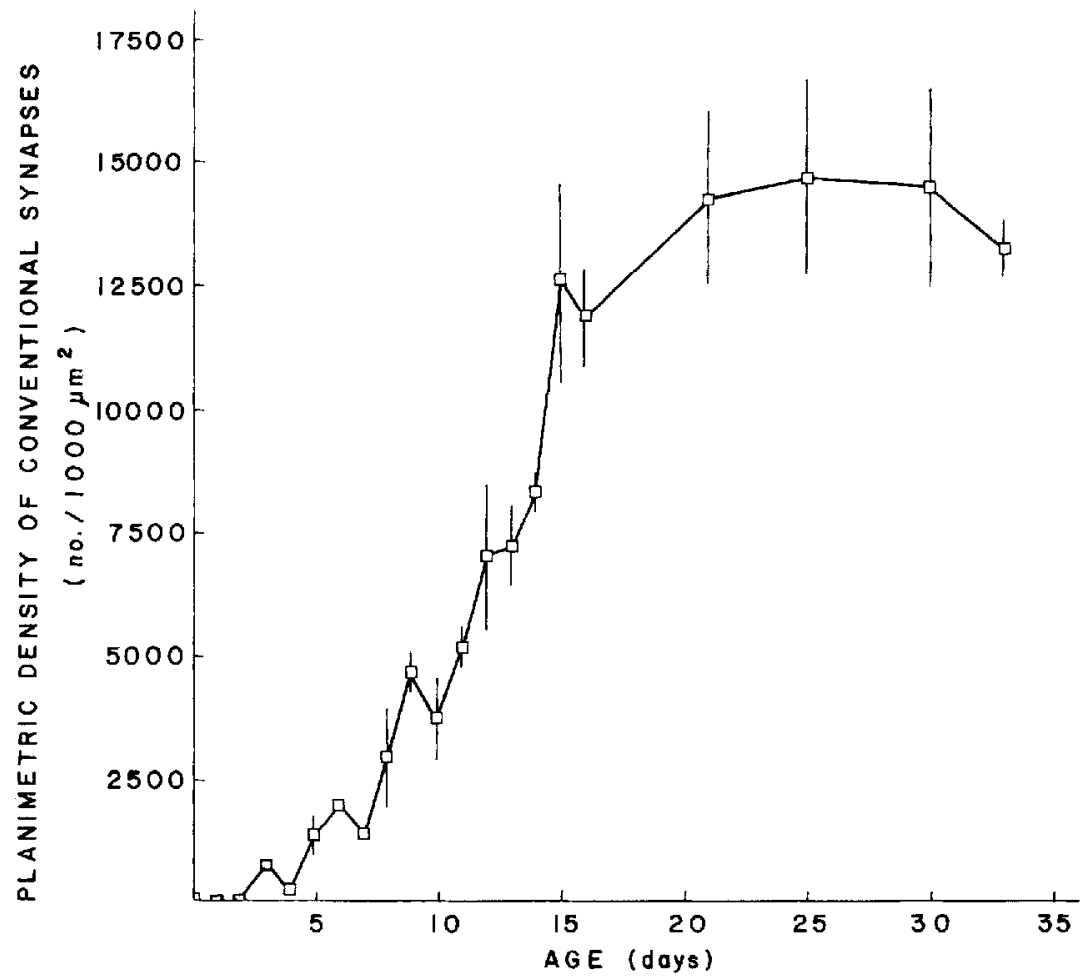

Fig. 14 Planimetric density of conventional synapses versus age. These data are the product of the numerical density of conventional synapses and the IPL thickness at the same age. 


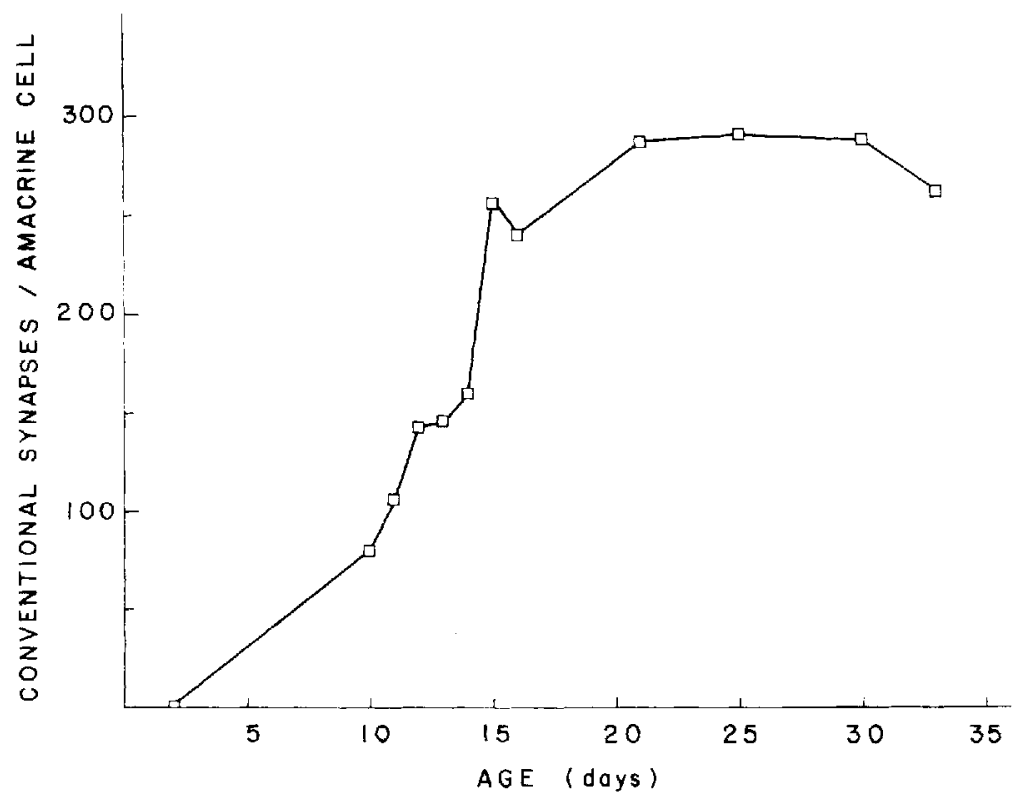

Fig. 15 Conventional synapses per amacrine or interplexiform nucleus. These data points are the mean planimetric density of conventional synapses at each age divided by the mean nuclear density of the amacrine plus interplexiform cells of the same age. The nuclear density is computed from a linear regression calculated by the method of least squares.

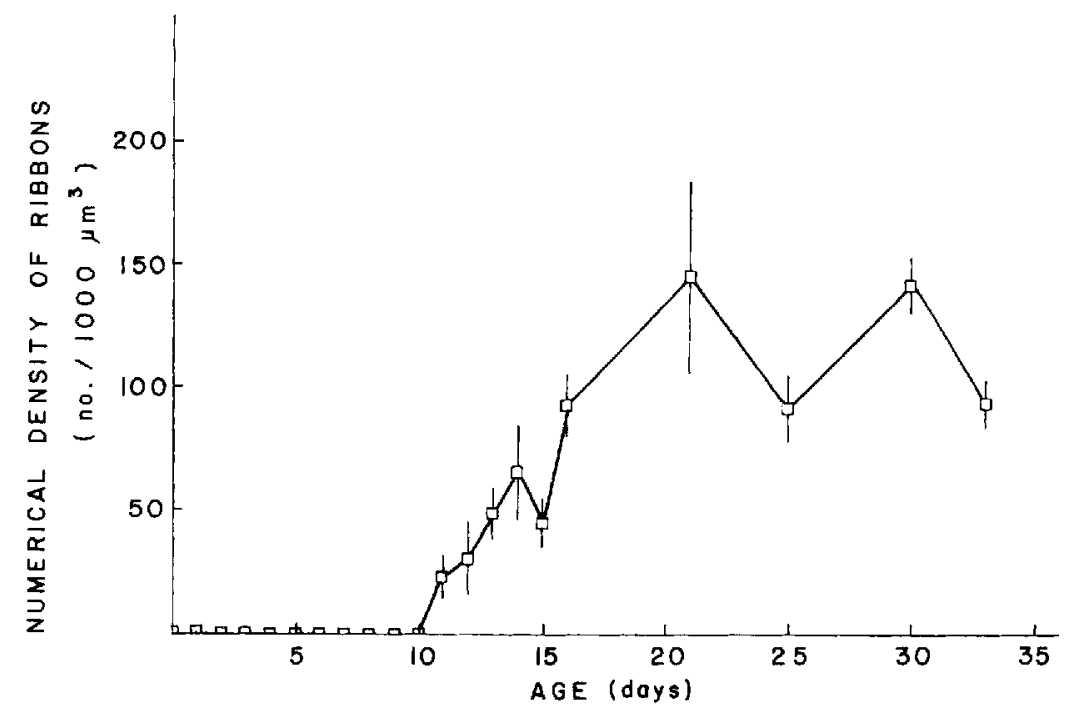

Fig. 16 Numerical density of ribbons versus postnatal age. Each point represents data from six animals on the average, with no point representing less than three animals.

apses $/ 1,000 \mu \mathrm{m}^{3}$ by day 10 (fig. 13). Retinal planimetric density increased to 3,686 synapses $/ 1,000 \mu \mathrm{m}^{3}$ (fig. 14). By day 10 there were 79 conventional synapses per amacrine plus interplexiform cell nucleus (fig. 15). Conventional synapses were produced at a mean rate of 0.41 synapses/nucleus/hour. Expressed another way, each amacrine or interplexiform 


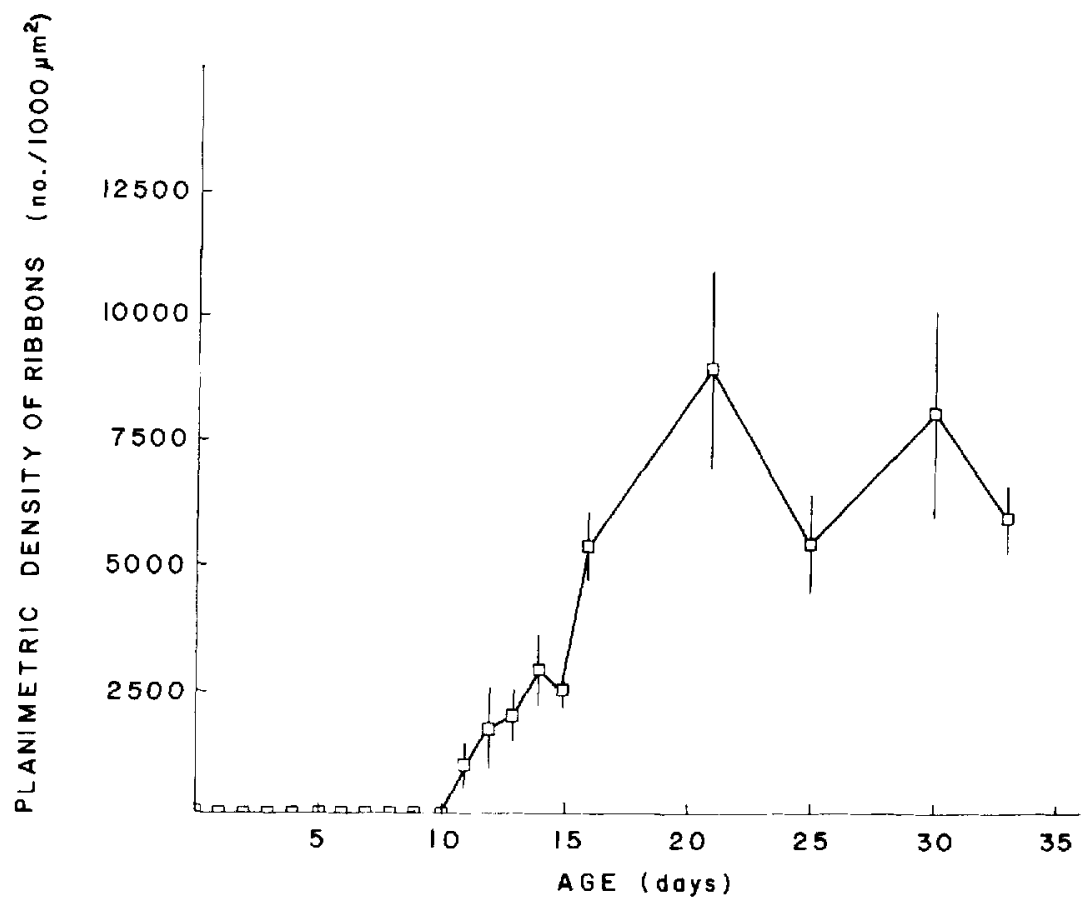

Fig. 17 Planimetric density of ribbons versus age. These data are the product of the numerical density of ribbons and the inner plexiform layer (IPL) thickness at the same age.

cell produced one new conventional synapse every 146 minutes during Phase I.

Phase II (day 11 to day 15) was characterized by the simultaneous rapid increase in ribbon and conventional synapses. The IPL continued to increase in thickness during this phase achieving a value of $60 \mu \mathrm{m}$ on day 15 . The numerical density of conventional synapses increased throughout this interval to 223 synapses $/ 1,000 \mu^{3}$ at day 15 (fig. 13). The rate of density increase, 1.15 synapses/ $1,000 \mu \mathrm{m}^{3} /$ hour, was nearly three times greater than that of Phase I. Retinal planimetric density of conventionals increased to 12,550 per $1,000 \mu \mathrm{m}^{2}$ at day 15 (fig. 14) and the mean number of conventionals per amacrine or interplexiform nucleus on that day was 252 (fig. 15), During Phase II conventionals increased at a mean rate of 1.4 synapses per nucleus per hour, or one new synapse per cell every 42 minutes.

The first ribbons in the IPL were seen on day 11 and increased in numerical density until day 14 (fig. 16). The slight drop in density on day 15 was not statistically significant ( $p>0.4$ ). The mean rate of increase in ribbon numerical density to the value of 45 ribbons/ $1,000 \mu \mathrm{m}^{3}$ on day 15 was 0.38 ribbons $/ 1,000$ $\mu \mathrm{m}^{3} /$ hour. Planimetric density of ribbons increased to 2,532 ribbons $/ 1,000 \mu \mathrm{m}^{2}$ on day 15 (fig. 17). The number of ribbons increased at a mean rate of 0.41 ribbons/bipolar nucleus/ hour to 49 ribbons/bipolar nucleus on day 15 (fig. 18). Thus each bipolar cell produced one new ribbon every 147 minutes.

Phase III (day 15 to day 33) was characterized by a sharp reduction in the rate at which both ribbon and conventional synapses were produced. During this phase each descriptive statistic reached its maximal value. Inner plexiform layer thickness stabilized at approximately $60 \mu \mathrm{m}$ in Phase III. Conventional synapses gradually increased to a numerical density of 250 synapses $/ 1,000 \mu^{3}$ (fig. 13) and a planimetric density of 14,450 synapses $/ 1,000 \mu \mathrm{m}^{2}$ (fig. 14). Each amacrine or interplexiform nucleus had a mean of 285 conventional synapses in Phase III (fig. 15).

The numerical density of ribbons fluctuated around a mean value of 113 ribbons $/ 1,000 \mu \mathrm{m}^{3}$ after day 16 (fig. 16). Planimetric density and the number of ribbons/bipolar nucleus also 


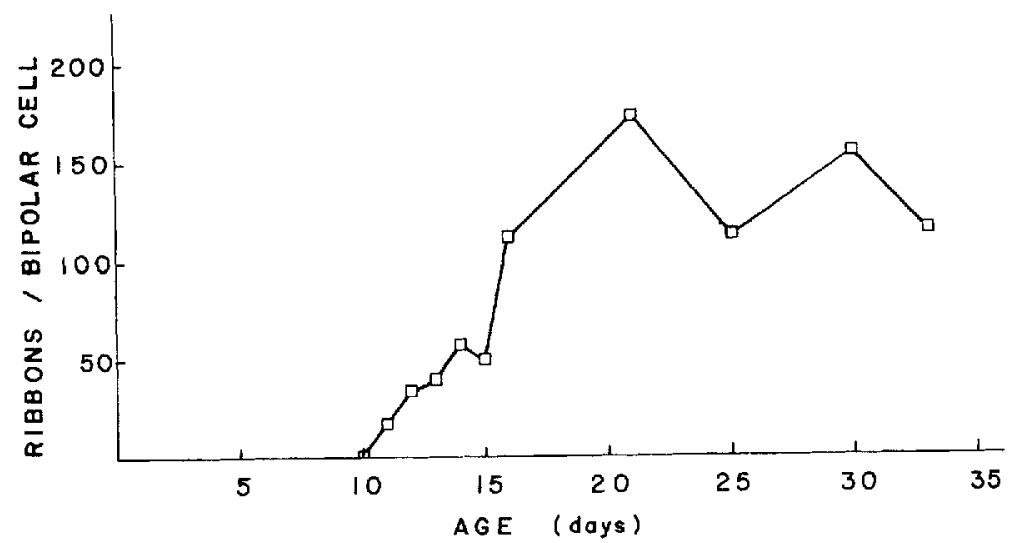

Fig. 18 Ribbons per bipolar nucleus versus age. These data points are the mean planimetric density of ribbons, divided by the mean nuclear density of bipolars. The nuclear density is computed from a linear regression calculated by the method of least squares.

fluctuated in Phase III. The mean number of ribbons per $1,000 \mu \mathrm{m}^{2}$ was 6,830 (fig. 17) while the mean number of ribbons/bipolar nucleus was 129 over the period from day 16 to day 33 (fig. 18).

The fraction of conventional synapses found in serial configuration was low at all ages. None were identified before day 10 and the maximal numerical density of eight synapses/ $1,000 \mu \mathrm{m}^{3}$ was achieved on day 30 . The planimetric densities of serial conventionals remained low with a mean value of 176 synapses/1,000 $\mu \mathrm{m}^{2}$ seen from day 10 to day 33 . Intersynaptic distance clearly affects the probability of intersecting a pair of conventional synapses, and thus this statistic underestimates the number of synapses in serial configuration. Nonetheless, the values found for mouse were much lower than those found for Xenopus (Fisher, '76).

\section{DISCUSSION}

These data demonstrate the existence of three rather distinct phases of development in the retinal IPL of the mouse. This discussion will deal with each of these phases in turn and then close with a quantitative comparison of this and other IPL's.

The most striking aspect of Phase $I$ is that synaptogenesis occurs in the absence of any sensory input. The lack of ribbon synapses suggests that bipolar signals do not reach the IPL, but the possibility remains that early bipolar synapses lack ribbons. Even so, the absence of sensory input may be inferred from several observations by others. First, the eyes are closed. Second, photoreceptor outer segments do not begin to develop until half-way through Phase I and then only in a few receptors (Olney, '68). Third, the bipolar dendrites have not yet invaginated the photoreceptor terminals (Blanks et al., '74). Fourth, the bipolar cells themselves have probably not made their terminal divisions (Sidman, '61).

During Phase II both types of synapses develop most rapidly. The ribbons, previously $a b-$ sent, increase in both size and number. The conventionals increase in number and vary in size. Interestingly, the decrease in the mean length of conventional synapses occurs at the time of the greatest surge in their numbers, which suggests that the new synapses are smaller than those already in existence. The size distributions (not shown here, but computed in the process of generating fig. 11) bear this out. Mean synaptic length was reduced as a result of the addition of smaller synapses to the extant distribution.

The onset of Phase II is not linked to any other obvious change in the animal's development, but the offset is synchronous with the opening of the eyes (Fisher, unpublished data). It is tempting to infer that visual stim. uli at eye opening inhibited synaptogenesis; this would account for the synchrony observed. But, since mice reared in darkness show a similar time course of both IPL development and eye opening (Fisher, in preparation), this explanation can be excluded. Moreover, in rabbit pups (McArdle et al., '77) 
TABLE 1

Comparative statistics for retinal synaptic arrays

\begin{tabular}{lccc}
\hline & $\begin{array}{c}\text { Adult } \\
\text { numerical } \\
\text { density } \\
\text { No./,000 } \\
\left.\mu \mathrm{m}^{3}\right)\end{array}$ & $\begin{array}{c}\text { Adult synapses } \\
\text { or ribbons } \\
\text { nucleus } \\
\text { (No./NL } \\
\text { nucleus })\end{array}$ & $\begin{array}{c}\text { Maximal } \\
\text { rate of } \\
\text { formation } \\
\text { (No.nucleus } \\
\text { hour) }\end{array}$ \\
\hline Ribbons & & & \\
$\begin{array}{l}\text { Goldfish } \\
\text { Xenopus }\end{array}$ & 17 & 18 & - \\
Mouse & 80 & 350 & 4.6 \\
Conventionals & 113 & 65 & 0.2 \\
Goldfish & 120 & 140 & \\
$\begin{array}{l}\text { Xenopus } \\
\text { Mouse }\end{array}$ & 600 & 2,800 & -7 \\
\hline
\end{tabular}

These data represent adult values of numerical density and of number of synapses/nucleus. To make the figures comparable, the number/nucleus for mouse are recalculated using bipolar plus amacrine plus interplexiform planimetric density as the denominator. Maximal rates of formation are computed on the same basis.

and goldfish (Fisher and Easter, '79) synaptogenesis continues even when the retina is illuminated.

One final point about Phase II concerns the bipolar cell. Blanks et al. ('74) has shown that the dendrites of the bipolars are invaginating the photoreceptor terminals at this time. Therefore, the bipolar cell is differentiating synaptically at both ends, simultaneously.

Phase III is characterized by no further developments in the central retina. This contrasts with the continued development in the retinas of adult amphibians (Xenopus laevis [Fisher, '76] and Rana pipiens [Fisher, '72]) and fish (Carassius auratus [Fisher and Easter, '791). One salient difference between the mouse on the one hand and the fish and amphibians on the other is that the retinas of the latter two continue to grow while the former does not.

Development of the mouse retina has been qualitatively described by Olney ('68). The quantitative morphological approach used in this report, however, is an outgrowth of the pioneering work of Dowling and Boycott ('66), who revealed the inherent simplicity of the IPL, and of Dowling ('68) and Dubin ('70), who examined this layer in different vertebrate species. The extra variable of ontogeny has been explored here and in earlier reports (Fisher, '72, '76; Fisher and Easter, '79), and it seems appropriate now to summarize and compare these developmental studies (table 1 ).

Several features warrant comment. First, in both numerical density and the number of synapses per nucleus, conventional synapses far exceed ribbons in all species. Second, mouse and goldfish have similar numbers of ribbons or conventionals per nucleus while the value for Xenopus is much higher. But, the ratio of conventionals per nucleus to ribbons per nucleus for goldfish and Xenopus are about equal while the same ratio for mouse is much lower. Third, the maximal rate of synapse formation in Xenopus is far greater than in mouse (values for goldfish were not obtained).

Cragg ('67,'72) reported the numbers of synapses per nucleus in cortical tissue. The values he gives, however, are the synapses on the neurons in question. The present data represent the mean number of synapses $b y$ the neurons. The quantitative statistics given in this paper are the only data of this sort available, and as such, they may be useful for comparison with similar work elsewhere in the nervous system, or in other species.

\section{ACKNOWLEDGMENTS}

I thank Sharon Burke for her invaluable technical assistance and Bonita Johnson for typing the manuscript. I especially appreciate the suggestions and assistance given by Stephen S. Easter, Jr. in the preparation of the manuscript. This work was supported by Research Grant EY-01281 from the National Eye Institute.

\section{LITERATURE CITED}

Abercrombie, M. 1946 Estimation of nuclear population from microtome sections. Anat. Rec., 94: 239-247.

Blanks, J. C., A. M. Adinolfi and R. N. Lolley 1974 Synaptogenesis in the photoreceptor terminal of the mouse retina. J. Comp. Neur., 156: 81-94.

Boycott, B. B., J. E. Dowling, S. K. Fisher, H. Kolb and A. M. Laties 1975 Interplexiform cells of the mammalian retina and their comparison with catecholamine-containing retinal cells. Proc. R. Soc. Lond. B., 191: 353-368.

Colonnier, M. 1964 The tangential organization of the visual cortex. J. Anat. (London), 98: 327-344.

Cragg, B. G. 1967 The density of synapses and neurones in the motor and visual areas of the cerebral cortex. $\mathbf{J}$. Anat, 101: 639-654.

1972 The development of cortical synapses dur. ing starvation in the rat. Brain, 95: 143-150.

Dowling, J. E. 1968 Synaptic organization of the frog retina: an electron microscopic analysis comparing the retinas of frogs and primates. Proc. R. Soc. B., 170: 205-228.

Dowling, J. E., and B. B. Boycott 1966 Organization of the primate retina: electron microscopy. Proc. R. Soc. B., 166: 80-111.

Dowling, J. E., B. Ehinger and W. L. Hedden 1976 The interplexiform cell: a new type of retinal neuron. Invest. Ophthalmol., 15: 916-926.

Dubin, M. W. 1970 The inner plexiform layer of the ver- 
tebrate retina: a quantitative and comparative electron microscopic analysis. J. Comp. Neur., 140: 479-506.

Fisher, L. J. 1972 Changes during maturation and metamorphosis in the synaptic organization of the tadpole retina inner plexiform layer. Nature, 235: 391-393.

1976 Synaptic arrays in the inner plexiform layer of the developing retina of Xenopus. Dev. Biol., 50: 402-412.

1979 Interplexiform cell of the mouse retina: a Golgi demonstration. Invest. Ophthalmol., 18: 521-523.

Fisher, L. J., and S. S. Easter, Jr. 1979 Retinal synaptic arrays: continuing development in the adult goldfish. J. Comp. Neur., 185: 373-380.

Kolb, H., and R. W. West 1977 Synaptic connections of the interplexiform cell in the retina of the cat. J. Neurocytol, 6: $155-170$.

McArdle, C. B., J. E. Dowling and R. H. Masland 1977 Development of outer segments and synapses in the rabbit retina. J. Comp. Neur., 175: 253-274.

Olney, J. W. 1968 An electron microscopic study of synapse formation, receptor outer segment development, and other aspects of developing mouse retina. Invest. Ophthalmol., 7: 250-268.

Polyak, S. 1957 The Vertebrate Visual System. Heinrich Kluver, ed. U. of Chicago Press, Chicago, Illinois.

Ramon y Cajal, S. 1972 The Structure of the Retina. S. A. Thorpe and M. Glickstein, translators. Charles C Thomas, Springfield, Illinois.

Raviola, G., and E. Raviola 1967 Light and electron microscopic observations on the inner plexiform layer of the rabbit retina. Am. J. Anat., 120: 403-426.

Sidman, R. L. 1961 Histogenesis of Mouse Retina Studied with Thymidine- $\mathrm{H}^{3}$. G. K. Smelser, ed. Academic Press, New York.

Weibel, E. R. 1969 Stereological principles for morpho. metry in electron microscopic cytology. Int. Rev. Cytol., 26: 235-302.

West, R. W. 1972 Thick sections of Epon. Stain Technol., 47: 201.

Witkovsky, P., and J. E. Dowling 1969 Synaptic relationships in the plexiform layers of carp retina. Z. Zellforsch., 100: $60-82$. 